\title{
Ultra-sensitive measurement of brain penetration mechanics and blood vessel rupture with microscale probes
}

Abdulmalik Obaid ${ }^{*}, Y u-W e i W^{2,3 *}$, Mina Hanna ${ }^{1 *}$,Omar Jáidar ${ }^{2}$,William Nix ${ }^{l}$, Jun Ding ${ }^{2 *}$, Nicholas Melosh ${ }^{\text {** }}$

${ }^{1}$ Department of Materials Science and Engineering, Stanford University

${ }^{2}$ Department of Neurosurgery, and Department of Neurology, Stanford University

${ }^{3}$ Institute of Molecular Biology, Academia Sinica, Taiwan

*These authors contributed equally to this work

**Correspondence: dingjun@stanford.edu andnmelosh@stanford.edu

\begin{abstract}
Microscale electrodes, on the order of 10-100 $\mu \mathrm{m}$, are rapidly becoming critical tools for neuroscience and brain-machine interfaces (BMIs) for their high channel counts and spatial resolution, yet the mechanical details of how probes at this scale insert into brain tissue are largely unknown. Here, we performed quantitative measurements of the force and compression mechanics together with real-time microscopy for in vivo insertion of a systematic series of microelectrode probes as a function of diameter (7.5-100 $\mu \mathrm{m}$ and rectangular Neuropixels) and tip geometry (flat, angled, and electrochemically sharpened). Results elucidated the role of tip geometry, surface forces, and mechanical scaling with diameter. Surprisingly, the insertion force post-pia penetration was constant with distance and did not depend on tip shape. Real-time microscopy revealed that at small enough lengthscales $(<25 \mu \mathrm{m})$, blood vessel rupture and bleeding during implantation could be entirely avoided. This appears to occur via vessel displacement, avoiding capture on the probe surface which led to elongation and tearing for larger probes. We propose a new, three-zone model to account for the probe size dependence of bleeding, and provide mechanistic guidance for probe design.
\end{abstract}

\section{Introduction}

Microelectrodes implanted into the brain are a critical component of new neuroprosthetic applications and brain-machine interfaces (BMIs), including high-density silicon probes (70 x 20 
$\mu \mathrm{m})^{1}$, syringe injectable electronics $(\sim 100 \mu \mathrm{m})^{2}$, shuttle delivery ${ }^{3}$, and microwire arrays $(<20 \mu \mathrm{m}$ per wire $)^{4,5}$. As these new probes become more widespread and new fabrication techniques become available, understanding the mechanics of brain penetration and insertion of devices in the 10-100 $\mu \mathrm{m}$ size scale is essential for balancing geometric design, materials strength and tissue damage $\mathrm{e}^{6,7}$. Appropriate optimization of mechanical insertion into the tissue is critical for success, as large devices can cause traumatic tissue damage and scar formation, while thin devices may buckle under the loads necessary to penetrate the outer pia membrane ${ }^{4,8,9}$.

Here, we performed systematic measurements of in vivo brain insertion forces and tissue compression for microscale probes of different diameters $(7.5-100 \mu \mathrm{m}$ cylindrical microwires and rectangular Neuropixels) and tip geometry to provide a rigorous mechanical framework for guiding probe design and understanding in this critical size regime. Microwires are a useful model system in that they are available in a wide variety of sizes with uniform surfaces and tip shapes, and have long been used for recording structures deep in the brain ${ }^{8-12}$. While the general expectation is that smaller wire diameters should yield lower damage, actual data for insertion mechanics is sparse. Prior investigations in the $10-100 \mu \mathrm{m}$ probe size range have been limited to a few different sizes with relatively low sensitivity measurement units. Initial tissue damage studies with lower-resolution instrumentation were inconclusive about size dependence, with no significant differences found between large (cross-sectional dimensions $\sim 200$ x $60 \mu \mathrm{m}$ ) and small (100-140 x $15 \mu \mathrm{m})$ devices ${ }^{13}$.

Measuring the mechanics of such ultra-small devices inserting into the brain, a soft, ultracompliant material, are highly challenging due to the large insertion displacements ( $\mathrm{mm}$ to $\mathrm{cm}$ ) before penetration, together with rapid and/or small force events present during insertion. Compromises typically have to be made either in force sensitivity or temporal resolution; many features may have been missed in previous studies simply due to instrumental limitations ${ }^{8,14-16}$. To address these challenges, we developed a high-performance force-displacement measurement system using a modified nanoindentation head as a force transducer. Nanoindentation transducers are among the fastest, most sensitive force-displacement systems available with $3 \mathrm{nN}$ force and 1 ms temporal sensitivity. However, these instruments are designed with maximum displacements on the order of hundreds of microns ${ }^{17}$, compared to the millimeters of compression required before tissue penetration. This large displacement range was achieved by integrating an entire iNano nanoindenter (Nanomechanics Inc.) measurement head onto a long-travel linear actuator, and 
measuring force and time with the nanoindenter in a zero-displacement mode while the actuator traveled at a constant velocity.

In combination with the mechanical measurements we visualized the probe inserting with realtime epifluorescence or two-photon microscopy. These measurements allowed direct visualization of how blood vessels failed during insertion, leading to the discovery of a probe size regime where vessel rupture can be avoided altogether. We propose a new three-zone model for blood vessel rupture to explain these observations, which predicts that sufficiently small probes will displace blood vessels.

\section{Results}

\section{Penetration force measurement}

A high-performance mechanical measurement system was developed to monitor the forces and displacements during penetration of soft $(\sim 1 \mathrm{kPa})$ brain tissue and mimics. This apparatus (Figure 1a) used a NanoMechanics iNano InForce 50 indentation head as the force transducer. The transducer position relative to the tissue is then controlled via a low-noise linear actuator moving $20 \mu \mathrm{m} / \mathrm{s}$ to a depth of $2.5 \mathrm{~mm}$. Tungsten microwire diameters of $7.5,15,25,35,50,80$, and 100 $\mu \mathrm{m}$ were measured, each with smooth sides. Different tip shapes (flat polished, angle polished, and electrosharpened) and tissue systems (phantom, ex vivo and in vivo) were prepared as described in the Methods section.

A schematic of the experimental apparatus and representative force-extension curves for both freshly excised ex-vivo (Fig. 1c,d) and in-vivo samples (Fig. 1e,f) is shown in Figure 1. As a 15 $\mu \mathrm{m}$ microwire extended at $20 \mu \mathrm{m} / \mathrm{s}$, the force increased exponentially for the first $\sim 250 \mu \mathrm{m}$, roughly $\sim 15$ times the diameter of the wire. Visually, as the wire is displaced the brain dimples without penetration, until a critical force is reached and the probe penetrates through the pia and a sudden drop in force is observed. This corresponded with the tissue visually relaxing around the wire, with the surface approaching its original location, which we interpret as indicating the microwire was inside the brain. From this penetration event both the force at puncture $\left(F_{p}\right)$ and the displacement to puncture $\left(d_{p}\right)$ were recorded.

Note that the force oscillations in vivo are not noise or stick-slip events, but instead the pulsatile effects from heartbeat and breathing (Fig. 1f). Both the curve shape and magnitude of the prepenetration phase are very similar between in vivo and ex vivo tests, which agrees with previous indentation measurements suggesting they have similar mechanical properties ${ }^{18-20}$. 


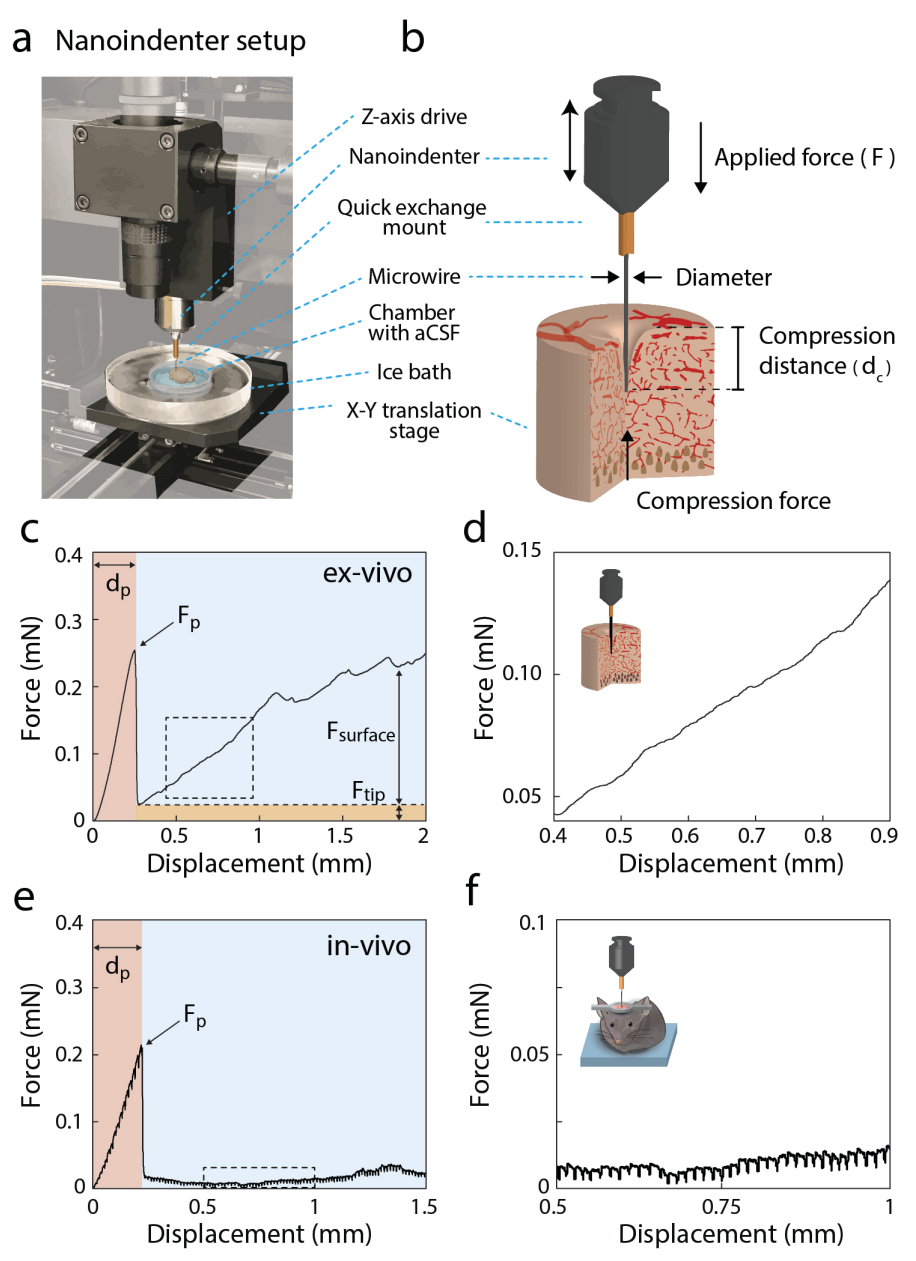

Figure 1 | Penetration of brain mimics, ex vivo and in vivo brain tissue

(a) Image of the adapted nanoindenter setup. Background electronics are grayed out to highlight areas of interest. The nanoindenter is mounted on a z-axis drive that provides the vertical translation. (b) Schematic illustration of insertion into brain tissue. (c,d) Force-displacement curve for insertion of $15 \mu \mathrm{m}$ diameter flat-polished wire into acutely excised brain tissue. The force increases exponentially pre-penetration, then increases linearly as a function of depth postpenetration. The linear increase in force is attributed to surfaces forces along the length of the wire $\left(F_{\text {surface }}\right)$ in addition to the constant force at the tip $\left(F_{t i p}\right)$. $(\mathbf{e}, \mathbf{f})$ Force-displacement curve for insertion of $15 \mu \mathrm{m}$ diameter flat-polished wires in vivo. Insertion into live brain tissue also displays a sharp drop in force during penetration, but the force plateaus as the wire is driven deeper, unlike the ex vivo case. Oscillations during insertion into live brain are consistent with breathing and heart rate.

Surprisingly, as the insertion force to drive the wire deeper into tissue in vivo after pia-penetration was constant as a function of depth. Slight features or humps are observed in the forcedisplacement curves, but without the monotonic linear force increases observed ex vivo. It is surprising that there is no increased resistance to insertion despite more probe area was in contact with the brain, yet this trend was highly consistent over dozens of experiments. We hypothesize this may be due to lubrication from active pumping of cerebrospinal fluid (CSF) or the dynamic 
motion from heartbeats preventing surface adhesion or static friction, yet more study needs to be done.

The absence of a linear increase in force post pia puncture in vivo shows that the pia is the primary mechanical barrier to electrode insertion; if a probe is stiff enough to penetrate the pia, it can be inserted to arbitrary depths. This suggests that initial penetration into brain tissue is the critical mechanical challenge in designing penetrating electrodes. Once implants are past the pia, there is minimal mechanical barrier to drive deeper into tissue; the force to insert $500 \mu \mathrm{m}$ deep is very similar to the force to insert two millimeters.

These penetration results were significantly different than for an agar phantom with similar elastic properties to the brain (Fig. S1). Agarose hydrogels are often used as mechanical brain mimics for indentation $^{15,16,21}$, yet during penetration distinct saw-tooth features were observed in the forcedisplacement curves at both $\mu \mathrm{m}$ and mm scales, unlike either ex vivo or in vivo cases. Thus, while useful for their elastic modulus, agar gels are not appropriate for penetration studies. 

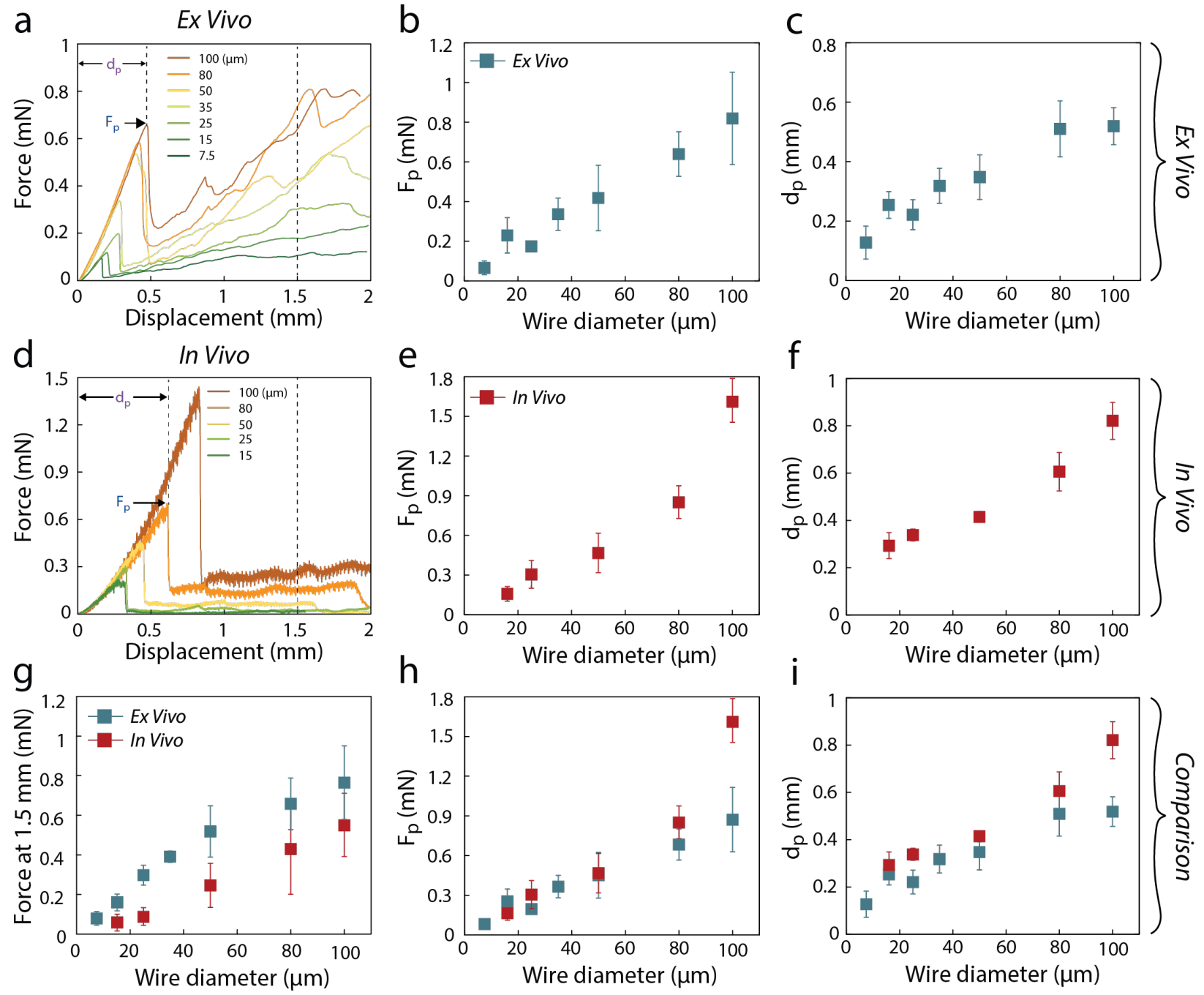

Figure 2 | Influence of Microwire Diameter

(a) Representative force-displacement curves comparing the response of different diameter flat-polished probes inserted into ex vivo brain at a rate of $20 \mu \mathrm{m} / \mathrm{s}$. Pia puncture occurs during abrupt drop in measured force, wherein the puncture force $\left(F_{p}\right)$ and displacement to puncture $\left(d_{p}\right)$ are extracted. (b) Relationship between puncture force and microwire is found to be linear. (c) The displacement to puncture as a function of wire diameter is also linear. (d) Representative force-displacement curves of flat-polished probes of increasing diameter inserted into live brain tissue at $20 \mu \mathrm{m} / \mathrm{s}$. (e,f) Relationship between puncture force and displacement to puncture as a function of size for insertion into live brain. (g) The force at $1.5 \mathrm{~mm}$ as a function of wire diameter for flat-polished wires, comparing ex vivo and in vivo. The forces after penetration are significantly lower in vivo than from ex vivo insertions. (h,i) Comparison of the scaling of puncture force and displacement to puncture with size between ex vivo and in vivo. Forces and displacements are found to be similar $50 \mu \mathrm{m}$ and below, with larger differences observed in vivo at large sizes.

\section{Influence of Microwire Diameter}

To understand the role of electrode size on brain insertion mechanics, the force-displacement to insert wires with diameters between $7.5 \mu \mathrm{m}$ to $100 \mu \mathrm{m}$ was measured ex vivo and in vivo, shown in Fig 2. All of these microwires had highly consistent, flat-tipped geometries by polishing the distal ends; tip shape dependence will be discussed below. The slope of the initial loading, 
indicating the compliance of the brain tissue, was only weakly dependent on wire diameter, with slightly lower compliances for smaller probes (Fig. S2). This suggests that over the range of the wire diameters studied, bulk brain tissue mechanics are generally homogeneous, consistent with previous studies on indentation into brain tissue $\mathrm{e}^{17,22}$.

The pia penetration event shape was highly stereotyped across all sizes, yet the force magnitudes to cause puncture $\left(F_{p}\right)$ varied by over an order of magnitude; from $185 \pm 40 \mu \mathrm{N}$ for $15 \mu \mathrm{m}$ wires, to $1610 \pm 239 \mu \mathrm{N}$ for $100 \mu \mathrm{m}$ diameter in vivo. Both the $F_{p}$ and compression $d_{p}$ scaled linearly with probe diameter, rather than the cross-sectional area of the tip. This observation is consistent with models of crack-initiated or energy-limited failure in compliant materials ${ }^{23,24}$, indicating the failure of the pia may occur via one of these modes. From this data, the force and compression needed to puncture the brain could be directly calculated based on the size of the wire in vivo:

$F_{p}(\mu N)=34.6+(10.07) * d(\mu m)$,

$d_{p}(\mathrm{~mm})=0.212+(5.06) * d(\mu \mathrm{m})$.

The insertion force past pia penetration was roughly constant for all in vivo tests (Fig. 2d), yet the magnitude of this force scaled linearly with diameter (Fig. 2e). Larger probes required larger absolute magnitudes of insertion force, yet did not increase significantly once through the pia. These observations are consistent with a friction model for in vivo tissue insertion, where the normal restoring force arises from lateral tissue displacement proportional to the probe's diameter. The linear relationship suggests the coefficient of friction is constant over all probe diameters studied. Interestingly, a significant difference between ex vivo and in vivo penetration force and compression exists for microwires of $100 \mu \mathrm{m}$ diameter $(\mathrm{p}<0.05$, two-way ANOVA, post hoc Bonferroni) (Fig. 2h,i). The significantly higher forces required for penetration in vivo for larger wires is unclear at the moment, yet may involve pressurization of the vasculature due to blood flow $^{25}$, or continual tissue movement due to breathing and heartbeat. 
a
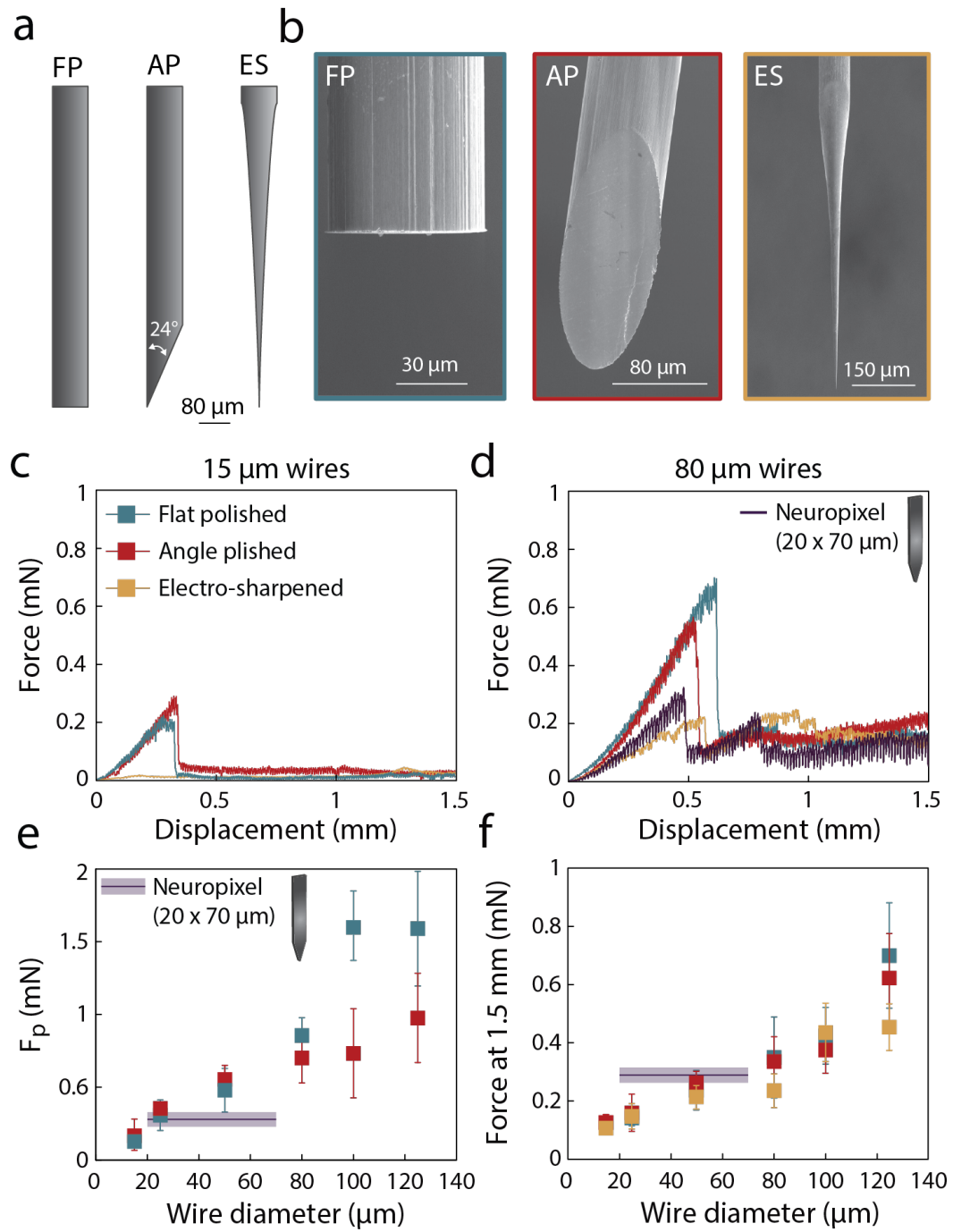

Figure 3 | Tip geometry dependence

(a) Graphical illustration of the three different tip geometries studied; flat-polished (FP), angle-polished (AP), and electrosharpened (ES). (b) Representative SEM images of the three different tip geometries. (c,d) In vivo forcedisplacement curves for 3 tip geometries for $15 \mu \mathrm{m}$ diameter (c) and $80 \mu \mathrm{m}$ diameter (d) microwires. A silicon probe (Neuropixel) with a $70 \mu \mathrm{m}$ wide, $20 \mu \mathrm{m}$ thick geometry is plotted in comparison with $80 \mu \mathrm{m}$ wires (d). (e) Measured puncture force in vivo as a function of wire diameter for FP and AP wires and a Neuropixel. Neuropixel values are plotted with wire diameter values between 20 and $70 \mu \mathrm{m}$ because of their rectangular cross-section. (f) In vivo force at $1.5 \mathrm{~mm}$ depth as a function of wire diameter for all tip geometries tested, including Neuropixels.

\section{Tip geometry dependence}

Sharpening the probe tip geometry is a common strategy for reducing tissue compression in penetrating microelectrodes ${ }^{26,27}$ which may result in less tissue damage, such as for Michigan-style probe arrays ${ }^{28,29}$. To elucidate the effects of probe shape in the $10-100 \mu \mathrm{m}$ range, penetration of angle-polished $\left(24^{\circ}\right)$ and electrosharpened probes (tip radius $\sim 10 \mathrm{~nm}$, Fig. $3 \mathrm{~b}$ ) were studied in comparison to the flat-polished tips. Two representative force-displacement curves for $15 \mu \mathrm{m}$ and $80 \mu \mathrm{m}$ wires of the three different tip geometries are shown in Figures $3 \mathrm{c}$ and $3 \mathrm{~d}$ for in vivo tests 
(ex vivo shown in Figure S3). Flat and angle-polished wires showed no statistically different behavior for microwires $\leq 100 \mu \mathrm{m}$ in diameter (Fig. 3e, Fig. S6), indicating at this lengthscale angle sharpening has no effect. Yet the results diverged $>100 \mu \mathrm{m}$, where the force and distance for angle-polished tips were lower than flat-polished tips. To further confirm this trend, microwires with $125 \mu \mathrm{m}$ diameter were tested, and $F p$ and $d p$ were again lower for angle-polished tips, in agreement with results for millimeter-scale needles ${ }^{30,31}$. This data suggests that our intuitive expectation that angled 'sharp' tips are better is true at macroscopic lengthscales, but breaks down in the $<100 \mu \mathrm{m}$ regime.

In contrast, electrosharpened wires displayed strikingly different behavior, with no discernable pia penetration event (Fig. 3c,d). There are numerous small rapid rises and drops in the force readout during insertion, but none could be classified as a distinct penetration of the brain surface. Synchronized videos of electrosharpened wire insertion also show no detectable insertion event, dimpling or tissue relaxation (Vid. S1). Hence, we cannot report a puncture force for electrosharpened wires. The forces during initial insertion into brain tissue were roughly an order of magnitude smaller than flat tips, ranging between 10 to $100 \mu \mathrm{N}$. Empirically, an electrosharpened tip that has been dulled or bent will still result in a distinguishable puncture event, albeit of much lower peak force magnitude than flat or angle polished microwires.

Interestingly, after pia puncture no insertion force difference was observed between flat, angled, or electrosharpened tips (Fig. 3f). This reveals that after penetration, the insertion force is dominated by surface forces along the shaft of the electrode and the surrounding brain tissue, rather than effects at the probe tip.

\section{Comparison to rectangular Neuropixel probes}

With the increasing prevalence of Michigan-style and Neuropixel silicon probes for high-density neural recording, we measured the mechanics of inserting rectangular 20x70 $\mu \mathrm{m}$ cross-section Neuropixels probes (Fig. 3e,f and Fig. S7). Neuropixels required pia penetration forces similar to that of $25 \mu \mathrm{m}$ diameter wires, which is close to the $20 \mu \mathrm{m}$ thickness of the probe tip (Fig. 3e). Past pia penetration, the insertion forces corresponded to cylindrical wires with diameters between 50 $\mu \mathrm{m}$ and $80 \mathrm{um}$, indicated by the purple bar in Figure 3f. Neuropixels have an equivalent surface area of a $\sim 57.5 \mu \mathrm{m}$ diameter wire, thus the insertion force appears dependent on circumference or surface area, rather than cross-sectional shape. These results are commensurate with the force independence on tip shape, indicating surface forces along the shaft of the probe dominate the bulk 
tissue insertion force, regardless of geometry. The effect of insertion speed was also studied using the Neuropixels, inserting at 2, 20 and $100 \mu \mathrm{m} / \mathrm{s}$ (Fig. S7g). No appreciable differences in the puncture force and compression were found. However, the rate of increase in force for the fastest speed $(100 \mu \mathrm{m} / \mathrm{s})$ was larger, likely due to the viscoelastic properties of brain tissue ${ }^{32}$. Finally, Neuropixels often exhibited a secondary 'peak' in the force curve after the pia penetration (Fig. $3 \mathrm{~d}$, purple trace). The origin of this event is unclear, but was quite common, observed in $\sim 80 \%$ of the force traces.

a

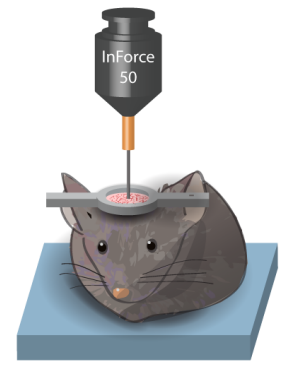

b

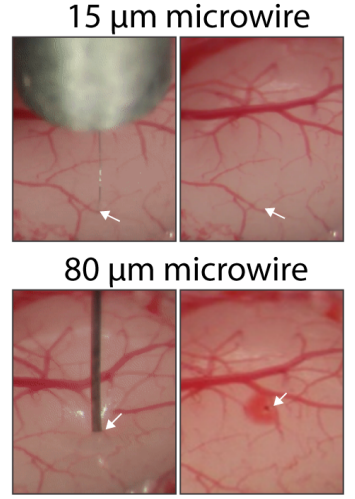

C

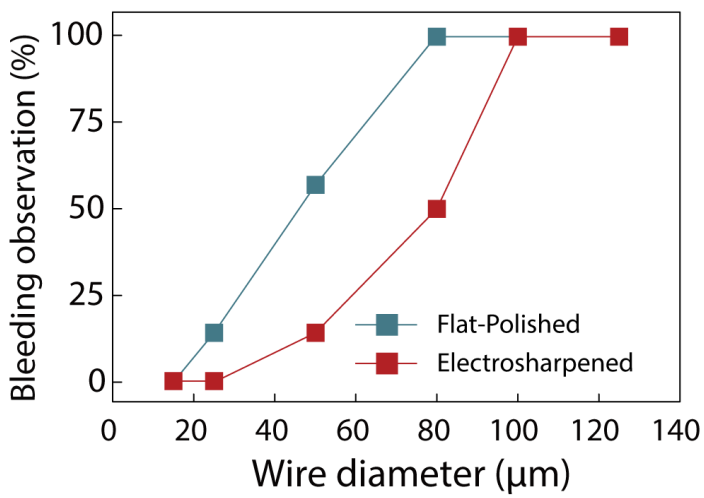

Figure 4| Surface bleeding observations

(a) Schematic of the in vivo force measurements. (b) Representative images of the surface of the brain before (left) and after (right) insertion of $15 \mu \mathrm{m}$ and $80 \mu \mathrm{m}$ microwires. No bleeding is observed is on the surface of the brain for $15 \mu \mathrm{m}$ wires. After retraction of the $80 \mu \mathrm{m}$ wire, the insertion site is marked by blood leaking from the surface. (c) Percent bleeding observed optically at the surface of the brain for insertion of flat-polished and electrosharpened wires. For insertion of $15 \mu \mathrm{m}$ wires, no bleeding was observed for all tip geometries. Between $25 \mu \mathrm{m}$ and $80 \mu \mathrm{m}$, electrosharpened wires showed fewer incidence of bleeding at the surface in comparison to flat-polished tips. For > $100 \mu \mathrm{m}$, all wires displayed bleeding at the surface. Large, visible vessels were avoided for all measurements.

\section{Effect of probe size on bleeding}

Bleeding during or post probe-insertion is a strong indicator of vascular and/or tissue damage, and can lead to serious subsequent trauma such as vasospasms. Larger probes, such as deep brain stimulation electrodes, always involve some level of hemorrhaging, yet probes in the sub-100 $\mu \mathrm{m}$ range have already shown different mechanical insertion behavior, thus may have different vessel damage as well. To quantify a bleeding event, we carefully observed with a magnified camera any surface bleeding, either during insertion or after the probe was withdrawn from the tissue, Fig 4a,b. These events were tallied on a binary yes/no basis, as the magnitude of hemorrhaging was difficult to accurately quantify, and all tests had an $\mathrm{N}>9$.

The results clearly show that the incidence of bleeding is strongly correlated with probe size, with a roughly sigmoidal dependence (Fig. 4c). From these observations, for $\geq 100 \mu \mathrm{m}$ probes there is 
always bleeding, transitioning to less common bleeding events between 100 to $25 \mu \mathrm{m}$ diameters, and finally to where no bleeding occurs for probes $<25 \mu \mathrm{m}$. Interestingly, electrosharpening the tip produces an offset in the curve rather than changing its overall shape, reducing the 'effective' wire diameter by 20-30 $\mu \mathrm{m}$ (Fig. 4c, red trace). Thus, bleeding can be reduced by selecting sharper tips, yet probe diameter is still the most important variable for hemorrhaging. For example, insertions for all $100 \mu \mathrm{m}$ tip shapes caused bleeding, but no bleeding for $15 \mu \mathrm{m}$ wires of any tip geometry. These results are highly surprising, suggesting it is possible to completely avoid vascular damage by going to small enough probe lengthscales. 

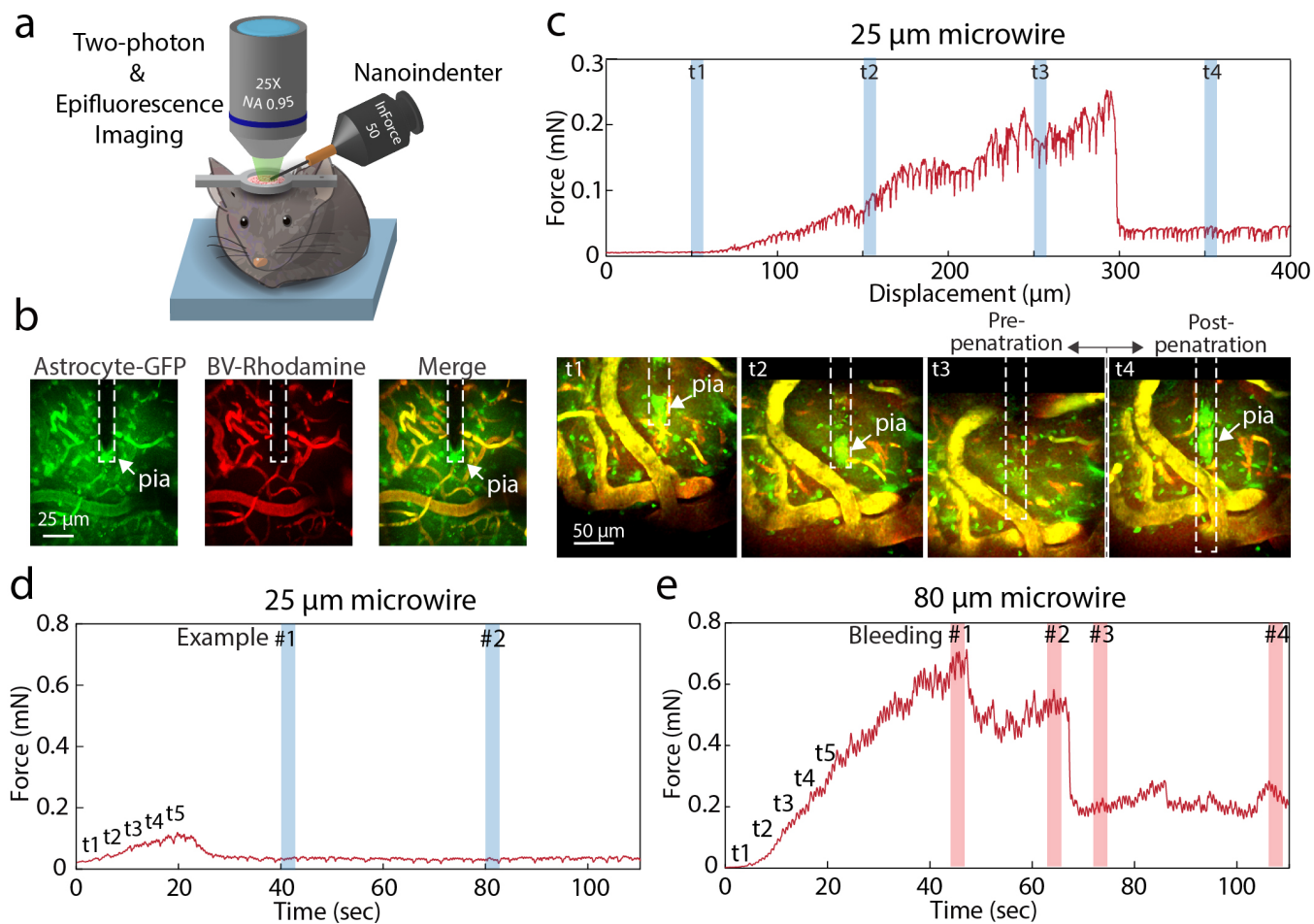

e
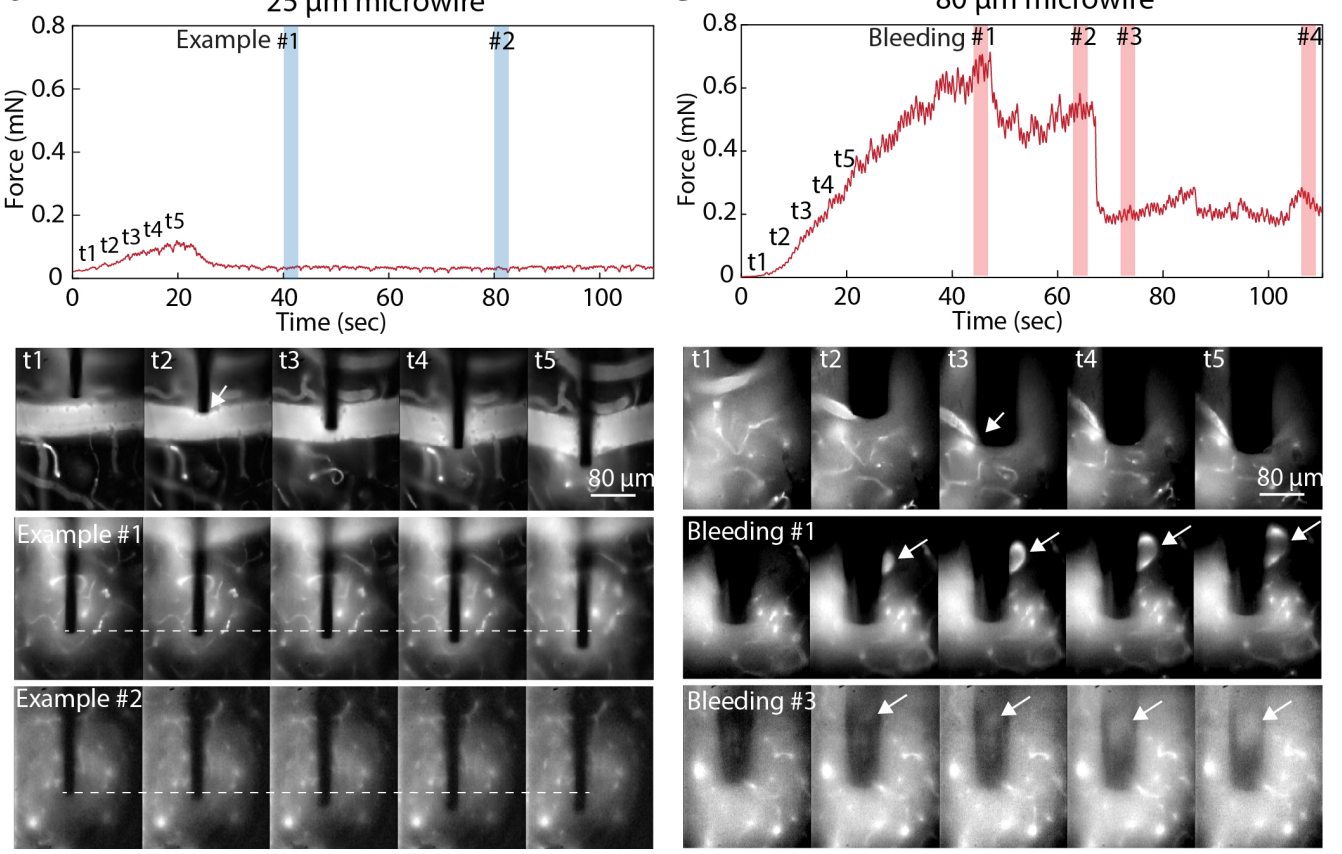

Figure 5 | Live two-photon and epifluorescence imaging with in situ force measurements

(a) Schematic of in vivo imaging with in situ force measurements. (b) eGFP-expressing astrocytes (Astrocyte-GFP) and blood vessels filled via tail vein injection of Rhodamine B (BV-Rhodamine) were imaged with two-photon microscopy. Image stacks were taken with sequential motion of the microwire. (c) Force vs displacement during the insertion of a $25 \mu \mathrm{m}$ microwire and the respective two-photon images at different steps in the insertion process. We observe the exponential increase in force during compression of the surface (t1-t3) as well as the lack of increase in force past penetration ( $t 4)$. Penetration was conformed with $2 \mathrm{P}$ imaging, showing the probe is past the pia at $t 3,4$. (d,e) Force-distance is plotted with synchronized epifluorescence videos of $25 \mu \mathrm{m}$ and $80 \mu \mathrm{m}$ microwire insertion, respectively. The exponential increase in force pre-penetration is correlated with the dimpling of the pia and surface vessels around the wire. During insertion of a small wire ( $25 \mu \mathrm{m}$, Fig. $6 \mathrm{~d}$ and Vid. S3), there is no noticeable bleeding or disruption of the vessels, even when the wire comes in contact with microvasculature. In contrast, the $80 \mu \mathrm{m}$ wire causes multiple bleeding events, both during the initial rupture of the pia surface and vessels beneath the surface.

\section{Monitoring insertion and blood vessel rupture with two-photon imaging and simultaneous}

\section{force/epifluorescence measurements}


To understand probe-tissue interaction during insertion and why bleeding may or may not occur, we modified the apparatus to also perform two-photon microscopy and real-time epifluorescence imaging during microwire insertion simultaneously with force (Fig. 5). These experiments were all performed in vivo with either 13, 25 or $80 \mu \mathrm{m}$ wires with a tail injection of a fluorescent dye (Rhodamine B, Fig. 5b) to aid the visualization of the vasculature network and bleeding events. Forces and tissue displacement were correlated by measuring force vs depth for a $25 \mu \mathrm{m}$ wire, pausing at certain locations to acquire two-photon (2P) image stacks (Fig 5b,c). No discernible changes were observed in the force measurements during the pauses to acquire the $2 \mathrm{P}$ image, suggesting the measurement technique did not alter normal insertion behavior. The location of the pia was identified by a sheath of astrocytes at the surface that is dragged deeper into the brain by the microwire insertion (Fig. 5b) ${ }^{33}$. Figure 5 c shows increasing force pre-pia penetration correlated with dimpling of the pia (visible at the tip of the probe) and compression of surface vessels/tissue around the wire, e.g. Fig 5c $t 1$ vs $t 2$. While the entire tissue appeared to displace vertically, only slight changes in the relative vasculature arrangement were observed. At a critical depth pia rupture occurred, which correlated most notably with the surrounding tissue relaxing vertically, e.g. Figure $5 \mathrm{c} t 3$ vs $t 4$. The pia was located mid-way along the microwire, rather than at the tip. In this image stack, no bleeding was observed at any depth.

We then took simultaneous real-time measurements of force and epifluorescence. During insertion of a $25 \mu \mathrm{m}$ wire at $20 \mu \mathrm{m} / \mathrm{s}$ (Fig. 5d, Vid. S2), there was no noticeable bleeding or disruption of the vasculature, despite the fact that in some cases the probe directly impacted a blood vessel. Timepoints $t 1-t 5$ in Fig $5 \mathrm{~d}$ show the probe push aside a $\sim 80 \mu \mathrm{m}$ blood vessel as the pia is compressed, yet no bleeding occurred even after pia penetration or deeper insertion. The subsequent timespan shows further insertion which also did not disrupt microvasculature in bulk tissue. Instead, this $25 \mu \mathrm{m}$ probe appeared to deflect the blood vessels to the side as it contacted them, without causing rupture.

This is in stark contrast to a large wire $(80 \mu \mathrm{m}$, Fig. 5e and Vid. S3), wherein significant disruption and bleeding is observed both at and post-pia penetration. During initial force loading prepenetration (timepoints $t 1-t 5$ ), the tissue is compressed and blood vessels were collapsed, but no visible bleeding occurred. Upon partial pia rupture the first bleeding event is visible at the surface, $\# 1$, and a hemorrhage is observed deeper in the tissue. Additional bleeding events \#2-4 during further insertion were also observed, highlighted in red. 
The videos show that blood vessel failure occurred by blood vessels getting trapped on the probe tip surface, then stretching as the probe was inserted further, then finally failing after significant elongation. Note that bleeding event \#1 in Figure 6e occurred by tearing the vessel at a location along the side of probe shaft, rather than at the tip. Thus, vessel rupture did not occur upon initial contact with the probe, nor likely due to a crack propagating near the tip, but only after being caught on the surface of the probe and stretched to failure. These rupture events often corresponded with features in the force profile, such as the sawtooth force profile for bleeding event \#4.

Next, we assessed the effect of insertion on the astrocytic populations and subpial vasculature with two-photon microscopy (Fig. S8, Vid. S4). A $13 \mu \mathrm{m}$ diameter microwire shows no disruption of the astrocytic populations or vasculature (blood brain barrier) during insertion, akin to the $25 \mu \mathrm{m}$ diameter microwire, while the $80 \mu \mathrm{m}$ microwire caused significant disruption of the local vasculature and astrocytic population. After retraction, a clear track of displaced astrocytes and bleeding vessels is left in place of the wire, indicating these larger probes caused significant disruption.

\section{Discussion}

\section{Three-Zone Blood Vessel Rupture Model}

The results show that smaller electrodes reduce rupture force, tissue compression, and likelihood of vessel rupture. Perhaps most interestingly, we observed a probe size range $<25 \mu \mathrm{m}$ that elicited no bleeding response. From video microscopy, these size wires pushed aside capillaries upon tip approach, allowing passage without causing rupture. For larger probes, blood vessel failure occurred by the vessels becoming captured at or near the probe tip, elongating with further insertion, and finally tearing or rupturing.

From these observations, we propose a three-zone conceptual model for the mechanism for blood vessel rupture (Fig. 6a). The model consists of a cylindrical penetrating probe of diameter $D$ encountering a blood vessel at some depth. Depending on the location of the vessel and the size of the probe, three distinct events may occur: (1) For vessels in the capture zone (red) located underneath the probe tip, blood vessels become entrapped on the surface of the probe, and are stretched as the probe continues to insert. The vessel finally fails due to the strain of elongation, rather than a cutting process near the tip, which often results in a vessel tear along the side of the probe, not underneath the probe itself. (2) In the displacement zone, vessels located in a thin ring neat the edge of the probe will instead be pushed aside, out from under the probe itself, avoiding 
capture on the surface. We speculate this occurs due to the differential lateral pressure near the edge of the probe tip, and the width of this zone is roughly constant, as it is a feature of the edge itself rather than the overall probe size. (3) Vessels in the deformation zone are compressed and distorted, but do not move significantly relative to the surrounding tissue.

We believe this model captures many of the observations made from the force and video evidence and particularly the scaling with probe size, Figure $7 \mathrm{~b}$. Large probes would have relatively little lateral displacement force underneath them, thus most of the region beneath the tip is a capture zone, with a thin band of displacement near the edge. As the probe size shrinks the displacement zone stays roughly constant, while the capture zone contracts. Finally, for a sufficiently small probe all vessels are displaced, rather than captured, allowing the probe to be inserted deeply into tissue without damaging the vasculature. Note that a small probe inserted directly on top of a very large blood vessel would still likely eventually cause penetration and bleeding, thus avoidance of large vessels should still be preferred.

These observations also open an interesting insights into how insertion velocity is important at different size scales. Multiple studies have found that injecting large probes very quickly $(>1 \mathrm{~m} / \mathrm{s})$ could reduce the amount of bleeding and tissue damage, ascribed to the stiffer nature of the viscoelastic tissue at these speeds. However, for the very small $<20 \mu \mathrm{m}$ probes which rely upon vessel displacement, it may be more beneficial to insert slowly ${ }^{34}$, allowing time for the vessels to move out of the way. Further velocity measurements are underway, which may shed more light on the insertion mechanics for these small probe dimensions. 


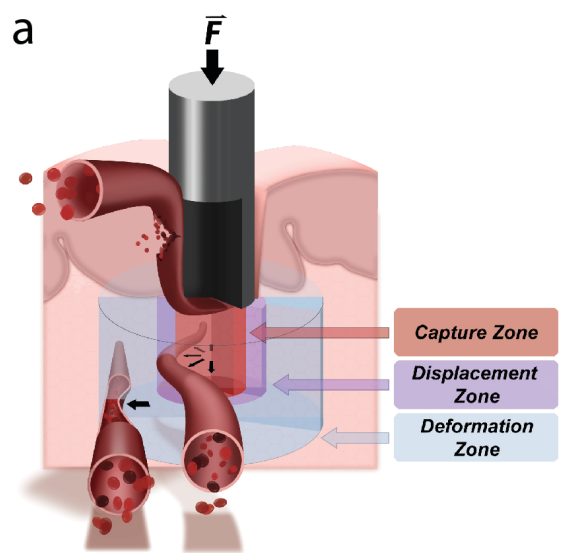

b
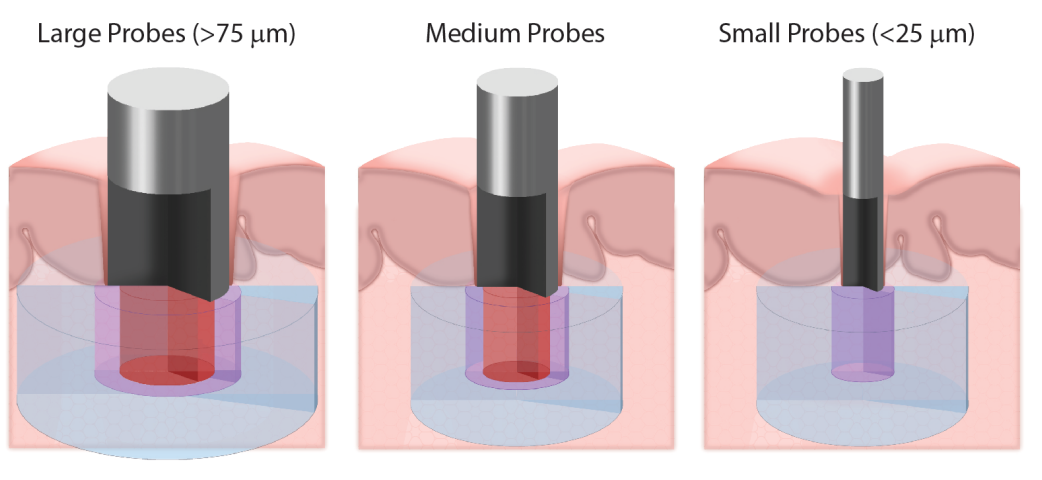

Figure 6 | Three Zone Blood Vessel Rupture Model and Probe Material Design Choices

(a) The three zone model for blood rupture mechanics consists of a penetrating probe encountering a blood vessel at some depth within the tissue. Depending on the distance of the vessel and the size of the probe, three distinct events may occur: capture and eventual failure, displacement from under the tip, or deformation away from the probe. (b) In the proposed model, the size of the displacement zone is roughly constant with diameter, such that at a critical size regime approximately two times the width of the displacement zone, no blood vessel rupture will occur.

\section{Conclusion}

The mechanics of inserting a foreign object into the brain is one of the critical issues for in vivo implantations. While numerous studies exist for clinical insertion of large, millimeter scale probes, ultra-small microwires and silicon probes with dimensions less than $100 \mu \mathrm{m}$ are becoming much more common, yet little is known about their insertion properties. Here, we measured insertion of a series of different size and tip shape probes with high spatial and temporal resolution for agar, ex vivo and in vivo systems. These careful mechanical insertion measurements provided a number of unexpected findings. First, the insertion force did not increase with depth in vivo after the pia penetration event, unlike previous ex vivo experiments. This implies that once through the pia layer, it would be possible to insert a probe to arbitrary depths without buckling, although it is possible that heterogeneities (e.g., white vs grey matter) could alter the trajectory of the wires.

Mechanically, both the pia penetration force and amount of tissue compression scaled linearly with probe diameter rather than cross-sectional area. The amount of tissue compression scaled as $\sim 4$ times the microwire diameter, with smaller probes requiring less force and causing less tissue compression. Interestingly, no statistical difference in pia penetration force or compression was observed between flat and angle-polished wire tips, while ultra-sharp electrosharpened tips had negligible pia penetration force for all wire diameters. Once inside the tissue however, the required insertion force scaled with circumference, independent of tip shape. This indicates that the sidewalls dictate the internal insertion force, while the tip shape is more relevant for pia 
penetration, especially at larger probe sizes. Interestingly, rectangular 20x70 $\mu \mathrm{m}$ Neuropixel probes penetrated the pia like a $20 \mu \mathrm{m}$ device, but inserted deeper into the tissue as a cylindrical wire with equivalent circumference.

Combined force measurements together with in situ epifluorescence and two-photon imaging revealed that blood vessel rupture appears to occur by a process of: (1) capture upon the probe tip, (2) elongation with further probe insertion, and (3) eventual rupture, usually not located at the probe tip. This implies that the vasculature does not fail due to encountering the tip nor a crack propagating near the tip surface, rather via attachment to the probe followed by stretching. Measurements of bleeding as a function of probe diameter discovered a size regime $<25 \mu \mathrm{m}$ where no bleeding with insertion was observed. These observations imply that by proper scaling of the probe, both tissue compression and blood vessel rupture can be avoided, which may lead to greatly improved outcomes.

The results presented here provide quantitative insights for properly scaling neural probe designs and better understanding brain mechanics during insertion of microscale devices. It is clear that the model system chosen can have a significant effect on the experimental result. From combined force and real-time measurements, we propose a tearing mechanism for blood vessel failure, which could be avoided for sufficiently small probes. Substantial additional work remains to uncover the details of these mechanisms and shed light on multiple probes inserting at one time and insertion velocity dependence. The number of surprising observations highlight the utility of basic quantitative measurements, and reveals there is still much to be discovered about interfacing artificial devices together with the brain.

\section{Methods}

\section{Instrumentation:}

We developed a high-performance mechanical measurement system. This apparatus, shown in Figure 1, used NanoMechanic's iNano InForce 50 indentation head as the force transducer. A displacement control protocol is used to fix the center plate of the iNano, while a low-noise linear actuator moves the indenter head $2.5 \mathrm{~mm}$ into the brain at $20 \mu \mathrm{m} / \mathrm{s}$. The surgical apparatus positions the iNano head above the tissue, then extends the indentation head to push the microwire into the material while measuring force and displacement. A custom program was written to automate force detection of the thin water layer maintained above the hydrogel/brain surface. This 
was done by vibrating the nanoindenter tip and detecting a phase angle change induced by contact with the water, amplified at the interface by the capillary force pulling onto the tip. This point is referenced to be zero. The system was then programmed to insert by a user specified amount into the hydrogel/brain by a speed capped at $50 \mu \mathrm{m} / \mathrm{s}$ to prevent damage to the tool and assure accurate force measurements (as dictated by the internal hardware speed of the feedback loop).

\section{Hydrogel Preparation}

An agarose $0.6 \%$ hydrogel was made and poured into glass vials. The concentration of the hydrogel was chosen based on literature findings to best match the elastic modulus of the brain ${ }^{6,35}$, done previously via nanoindentation. ${ }^{17}$ Microwires inserted into the hydrogel were not easily cleaned, and typically disposed of consequently. Attempts were made by placing in boiling water, but subsequent insertions in fresh agarose solutions did not show repeating behavior. Using microwires freshly etched via oxygen plasma, the behavior was very consistent. During insertion experiments, water was added above the hydrogel solution to ensure hydration during the length of the test.

\section{Probe Fabrication}

Tungsten wires of varying diameters $(7.5,15,25,35,50,80,100 \mu \mathrm{m})$ were spooled, coated with Parylene $\mathrm{C}(\mathrm{PaC})$, and subsequently cut into 1" segments. Briefly, aggregates of microwires were placed into glass tubes, infiltrated with Apeizon black wax W, and subsequently polished to accomplish the desired tip angles (flat and $24^{\circ}$ ) and then released. Stainless steel rods with a 150 $\mu \mathrm{m}$ inter-diameter (ID) bore were cut via electron discharge machining (EDM) to ensure no burr existed after the cut. The parylene coating also acted to increase microwire diameter, allowing for each microwire to be coated with the needed amount to have a final OD of $140 \mu \mathrm{m}$, greatly reducing the deviation of each microwire as it is inserted into the $150 \mu \mathrm{m}$ diameter bore of the stainless-steel rods. Once inserted into the rods, each wire was glued in place using EpoTek 301. Etching in oxygen plasma etched the $\mathrm{PaC}$ to expose a length of microwire but kept the $\mathrm{PaC}$ embedded in the stainless-steel tube. This method allowed for consistent minimization of angular deviation, as the nanoindentation head can only measure force in the z-axis. For electrosharpened wires, bare tungsten wires were individually submerged in $0.9 \mathrm{M} \mathrm{KOH}$. 2V was applied between the wire and a Pt wire counter electrode using a Keithley 2600 SMU and current was recorded. Etching stopped when the submerged part of the wire broke away from the rest of the wire. This break is observed visually by use of a stereoscope and confirmed by a sharp decrease in current 
between the two electrodes. Sharpened tips were immediately cleaned with deionized water and isopropyl alcohol and stored for safekeeping.

Every microwire produced was imaged via SEM and documented for quality and reproducibility after manufacturing and each insertion. Cleaning of the tips post insertion was done in enzymatic soap, followed by acetone, isopropanol, ethanol, DI water, and PBS.

Animal and ex vivo brain excision

Adult (10 month) C57BL/6J mice (JAX\# 000664) were used for this study. All procedures were approved by Stanford University's Administrative Panel on Laboratory Animal Care. Animals were anesthetized with isoflurane and decapitated. The brain was exposed and chilled with icecold artificial CSF (aCSF) containing $125 \mathrm{mM} \mathrm{NaCl}, 2.5 \mathrm{mM} \mathrm{KCl}, 2 \mathrm{mM} \mathrm{CaCl} 2,1.25 \mathrm{mM}$ $\mathrm{NaH}_{2} \mathrm{PO}_{4}, 1 \mathrm{mM} \mathrm{MgCl} 2,25 \mathrm{mM} \mathrm{NaHCO}$, and $15 \mathrm{mM}$ D-glucose. Freshly excised mouse brains were maintained in ice-cold aCSF, and all measurement was done within 1 hour after excision. The mechanical properties of excised brain have been found to remain constant within one hour post-mortem as long as temperature is controlled ${ }^{18,20}$. Repeated measurements using a $25 \mu \mathrm{m}$ diameter wire showed deterioration of force required to insert past the pia beyond 1 hour after excision. As such, use of a quick-exchange system was developed to allow for as many insertions as possible within one hour of beginning the ex vivo preparation. Excised brain was stuck on a petri dish with medical grade cyanoacrylate and filled with aCSF cooled externally in an ice bath (Fig. 1). Surface blood vessels were avoided when possible. Prior to each insertion tests, a thin layer of aCSF was applied to the brain surface to prevent drying. Insertions were all into the motor and sensory cortex areas. Between six to eight insertions were done on each brain, and the position of each subsequent insertion was shifted by $500 \mu \mathrm{m}$. The nanoindenter was positioned $\sim 200 \mu \mathrm{m}$ above the surface of the brain and inserted at $20 \mu \mathrm{m} / \mathrm{s}$ to a depth of $\sim 2-2.5 \mathrm{~mm}$. The nanoindenter was pulled out rapidly $(1 \mathrm{~mm} / \mathrm{s})$ to speed up each insertion and consequently how many wires we could test per brain within 1 hour after excision. Wires were cleaned with enzymatic soap and isopropyl alcohol after each insertion to ensure all tissue residue is removed. To ensure reliability of measurements, subsequent brains tests altered order of diameters used. No clear difference in pia puncture behavior was shown between the two sequential orders tested in the time allotted Fig. S4), suggesting the mechanical properties of the brain tissue remained stable through the recording periods ${ }^{17}$. The large spread seen in these measurements is likely due to variations in blood vessel density or laminar structures of the mouse cortex. 


\section{$\underline{\text { In vivo force measurements }}$}

Mice were anaesthetized with an intraperitoneal injection of $17 \mathrm{mg} / \mathrm{ml} \mathrm{ketamine} \mathrm{and} 1.7 \mathrm{mg} / \mathrm{ml}$ xylazine in saline and positioned in a stereotaxic frame. Two bone screws were placed on the skull to provide mechanical stability. A head-plate was centered to the intended surgery site on the right hemisphere and fixed to the bone screws and skull with C\&B METABOND Cement (Parkell Inc.). Small (2-3 mm) craniotomies were made over the somatomotor and somatosensory cortices using standard procedures to expose the brain surface ${ }^{36}$. Ringer's solution was applied on the brain surface at all times to prevent tissue drying which causes bleeding. Dura mater was carefully removed with fine forceps to expose the pia surface. Mice were then transferred to the experimental apparatus for force measurements under anaesthetized condition.

In vivo two-photon and epifluorescence imaging

In vivo imaging were performed in adult (4-6 months old) Tg(Aldh111-EGFP,-DTA)D8Rth/J mice (JAX\# 026033), wherein the astrocytes express green fluorescent protein, eGFP. To reveal the blood vessels, a red fluorescent dye, Rhodamine B isothiocyanate-dextran (70kDa) solution (100 $\mu 1 ; 100 \mathrm{mg} / \mathrm{mL}$, Sigma, R9379), was injected through the tail vein. The mouse was mounted to an experimental apparatus on a motorized stage under the microscope (objective: XLUMPLFLN20xW; Olympus BW51, Japan). The nanoindenter and the microwires were mounted to a micromanipulator (MP-285, Sutter, USA) with an estimated 20 degree angle (Fig. 6a). Two-photon imaging was performed with a custom built 2-photon laser-scanning microscope equipped with a mode-locked Ti:sapphire laser Mai Tai eHP (Spectra-Physics, USA) ${ }^{37}$. Rhodamine B and eGFP were excited at $830 \mathrm{~nm}$ and $925 \mathrm{~nm}$ light wavelengths, respectively. Three-dimensional image stacks were taken every $50-100 \mu \mathrm{m}$ advances of the microwire. Epifluorescence imaging was performed with an arc lamp and appropriate filter sets (540-580 nm for excitation; 600-640 nm for emission) to monitor the fluorescence of Rhodamine B. A CCD video camera (XC-77, Hamamatsu, Japan) was used to acquire the video at 30 frames per second.

\section{References}

1. Jun, J. J. et al. Fully integrated silicon probes for high-density recording of neural activity. Nature (2017). doi:10.1038/nature24636

2. Liu, J. et al. Syringe-injectable electronics. Nat. Nanotechnol. 10, 629-635 (2015). 
3. Kozai, T. D. Y. \& Kipke, D. R. Insertion shuttle with carboxyl terminated self-assembled monolayer coatings for implanting flexible polymer neural probes in the brain. $J$. Neurosci. Methods 184, 199-205 (2009).

4. Du, Z. J. et al. Ultrasoft microwire neural electrodes improve chronic tissue integration. Acta Biomater. 53, 46-58 (2017).

5. Rivnay, J., Wang, H., Fenno, L., Deisseroth, K. \& Malliaras, G. G. Next-generation probes, particles, and proteins for neural interfacing. Science Advances 3, (2017).

6. Lecomte, A., Descamps, E. \& Bergaud, C. A review on mechanical considerations for chronically-implanted neural probes. Journal of Neural Engineering 15, (2018).

7. Chen, R., Canales, A. \& Anikeeva, P. Neural recording and modulation technologies. Nat. Rev. Mater. 2, 16093 (2017).

8. Yoshida Kozai, T. D. et al. Ultrasmall implantable composite microelectrodes with bioactive surfaces for chronic neural interfaces. Nat. Mater. 11, 1065-1073 (2012).

9. Patel, P. R. et al. Chronic in vivo stability assessment of carbon fiber microelectrode arrays. J. Neural Eng. 13, (2016).

10. Podder, P. K., Mallick, D., Samajdar, D. P. \& Bhattacharyya, A. Design, Simulation and Study of MEMS Based Micro-needles and Micro-pump for Biomedical Applications. Des. Simul. Study MEMS Based Micro-needles Micro-pump Biomed. Appl. 1-3 (2011).

11. Dotson, N. M., Hoffman, S. J., Goodell, B. \& Gray, C. M. A Large-Scale Semi-Chronic Microdrive Recording System for Non-Human Primates. Neuron 96, 769-782.e2 (2017).

12. Nicolelis, M. A. L. et al. Chronic, multisite, multielectrode recordings in macaque monkeys. Proc. Natl. Acad. Sci. 100, 11041-11046 (2003).

13. Szarowski, D. H. \& Shain, W. Brain responses to micro-machined silicon devices. Brain Res. 1-13 (2003).

14. Sharp, A. A., Ortega, A. M., Restrepo, D., Curran-Everett, D. \& Gall, K. In Vivo Penetration Mechanics and Mechanical Properties of Mouse Brain Tissue at Micrometer Scales. IEEE Trans. Biomed. Eng. 56, 45-53 (2009). 
15. Casanova, F., Carney, P. R. \& Sarntinoranont, M. In vivo evaluation of needle force and friction stress during insertion at varying insertion speed into the brain. J. Neurosci. Methods 237, 79-89 (2014).

16. Apollo, N. V. et al. Development and Characterization of a Sucrose Microneedle Neural Electrode Delivery System. Adv. Biosyst. 1700187 (2017). doi:10.1002/adbi.201700187

17. Budday, S. et al. Mechanical properties of gray and white matter brain tissue by indentation. J. Mech. Behav. Biomed. Mater. 46, 318-330 (2015).

18. Gefen, A. \& Margulies, S. S. Are in vivo and in situ brain tissues mechanically similar? $J$. Biomech. 37, 1339-1352 (2004).

19. Guertler, C. A. et al. Mechanical properties of porcine brain tissue in vivo and ex vivo estimated by MR elastography. J. Biomech. (2018). doi:10.1016/j.jbiomech.2018.01.016

20. Liu, Y.-L. et al. In vivo and ex vivo elastic properties of brain tissues measured with ultrasound elastography. J. Mech. Behav. Biomed. Mater. 83, 120-125 (2018).

21. Deepthi, R., Bhargavi, R., Jagadeesh, K. \& Vijaya, M. S. Rheometric Studies on Agarose Gel- A Brain Mimic Material. Sastech 9, 27-30 (2010).

22. MacManus, D. B., Pierrat, B., Murphy, J. G. \& Gilchrist, M. D. Mechanical characterization of the P56 mouse brain under large-deformation dynamic indentation. Sci. Rep. 6, 21569 (2016).

23. Fakhouri, S., Hutchens, S. B. \& Crosby, A. J. Puncture mechanics of soft solids. Soft Matter 11, 4723-4730 (2015).

24. Shergold, O. A. \& Fleck, N. A. Mechanisms of deep penetration of soft solids, with application to the injection and wounding of skin. Proc. R. Soc. A Math. Phys. Eng. Sci. 460, 3037-3058 (2004).

25. Kozai, T. D. Y. et al. Reduction of neurovascular damage resulting from microelectrode insertion into the cerebral cortex using in vivo two-photon mapping. J. Neural Eng. 7, (2010).

26. Jensen, W., Yoshida, K. \& Hofmann, U. G. In-vivo implant mechanics of flexible, silicon- 
based ACREO microelectrode arrays in rat cerebral cortex. IEEE Trans. Biomed. Eng. 53, 934-940 (2006).

27. Foggiato, J. Chemical Vapor Deposition of Silicon Dioxide Films. in Handbook of Thin Film Deposition Processes and Techniques (2009). doi:10.1016/b978-0815514428.50008-0

28. Fekete, Z., Németh, A., Márton, G., Ulbert, I. \& Pongrácz, A. Experimental study on the mechanical interaction between silicon neural microprobes and rat dura mater during insertion. J. Mater. Sci. Mater. Med. (2015). doi:10.1007/s10856-015-5401-y

29. Fiáth, R. et al. A novel multisite silicon probe for laminar neural recordings. in Procedia Computer Science (2011). doi:10.1016/j.procs.2011.09.015

30. Sharp, A. A., Restrepo, D. \& Gall, K. In Vivo Penetration Mechanics and Mechanical Properties of Mouse Brain Tissue at Micrometer Scale. Trans Biomed Eng (2009). doi:10.1109/TBME.2008.2003261.In

31. Bjornsson, C. S. et al. Effects of insertion conditions on tissue strain and vascular damage during neuroprosthetic device insertion. J. Neural Eng. 3, 196-207 (2006).

32. Rashid, B., Destrade, M. \& Gilchrist, M. D. Mechanical characterization of brain tissue in tension at dynamic strain rates. J. Mech. Behav. Biomed. Mater. (2014). doi:10.1016/j.jmbbm.2012.07.015

33. Miller, S. J. et al. Molecularly defined cortical astroglia subpopulation modulates neurons via secretion of Norrin. Nat. Neurosci. (2019). doi:10.1038/s41593-019-0366-7

34. Fiáth, R. et al. Slow insertion of silicon probes improves the quality of acute neuronal recordings. Sci. Rep. (2019). doi:10.1038/s41598-018-36816-z

35. Chen, Z.-J. et al. A realistic brain tissue phantom for intraparenchymal infusion studies. $J$. Neurosurg. 101, 314-322 (2004).

36. Dombeck, D. A., Khabbaz, A. N., Collman, F., Adelman, T. L. \& Tank, D. W. Imaging Large-Scale Neural Activity with Cellular Resolution in Awake, Mobile Mice. Neuron (2007). doi:10.1016/j.neuron.2007.08.003 
37. Carter, A. G. \& Sabatini, B. L. State-dependent calcium signaling in dendritic spines of striatal medium spiny neurons. Neuron (2004). doi:10.1016/j.neuron.2004.10.013

38. Lind, G., Linsmeier, C. E., Thelin, J. \& Schouenborg, J. Gelatine-embedded electrodes - A novel biocompatible vehicle allowing implantation of highly flexible microelectrodes. $J$. Neural Eng. 7, 046005 (2010).

39. Wester, B. A., Lee, R. H. \& LaPlaca, M. C. Development and characterization of in vivo flexible electrodes compatible with large tissue displacements. J. Neural Eng. 6, 024002 (2009).

40. Jorfi, M., Skousen, J. L., Weder, C. \& Capadona, J. R. Progress towards biocompatible intracortical microelectrodes for neural interfacing applications. J. Neural Eng. 12, 011001 (2015). 


\section{Supplementary Figures}

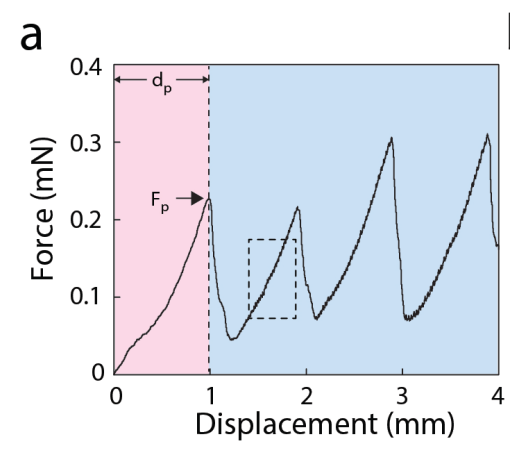

C

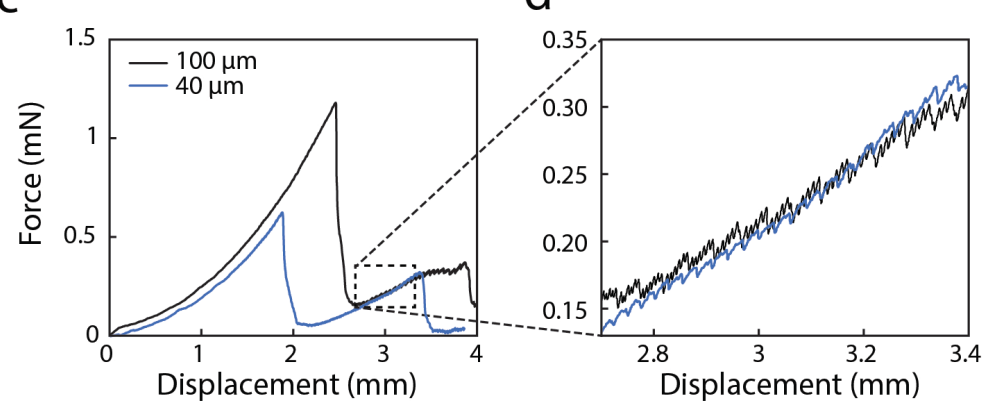

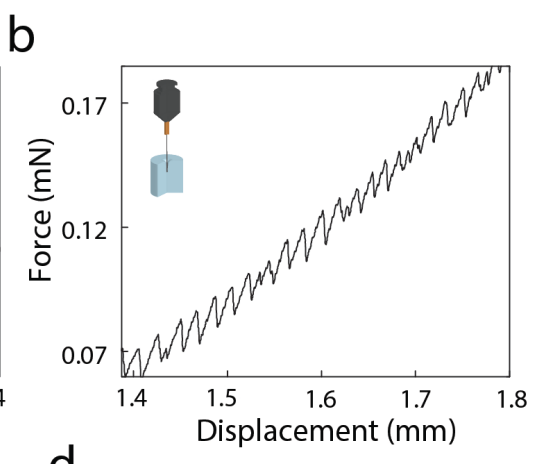

d

\section{(1)}

Figure S1 | Penetration mechanics and size-dependence for agarose brain mimics 
a

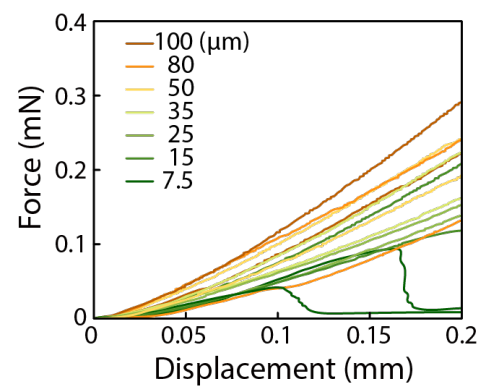

b

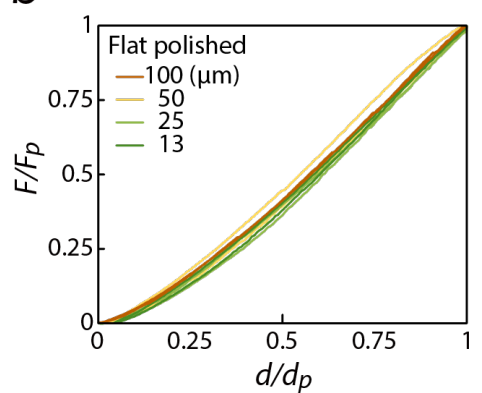

Figure S2 | Pre-penetration curves for flat-polished wires.

(a) Two representative raw traces of each size are shown. Coarse differences are found between larger and smaller wires in the force-displacement behavior pre-penetration. (b) Pre-penetration curves for flat-polished wires, two of each size, normalized by the force and depth at puncture. No trend with size is observed, suggesting that the underlying mechanics of indenting into brain tissue is similar between $10-100 \mu \mathrm{m}$. 

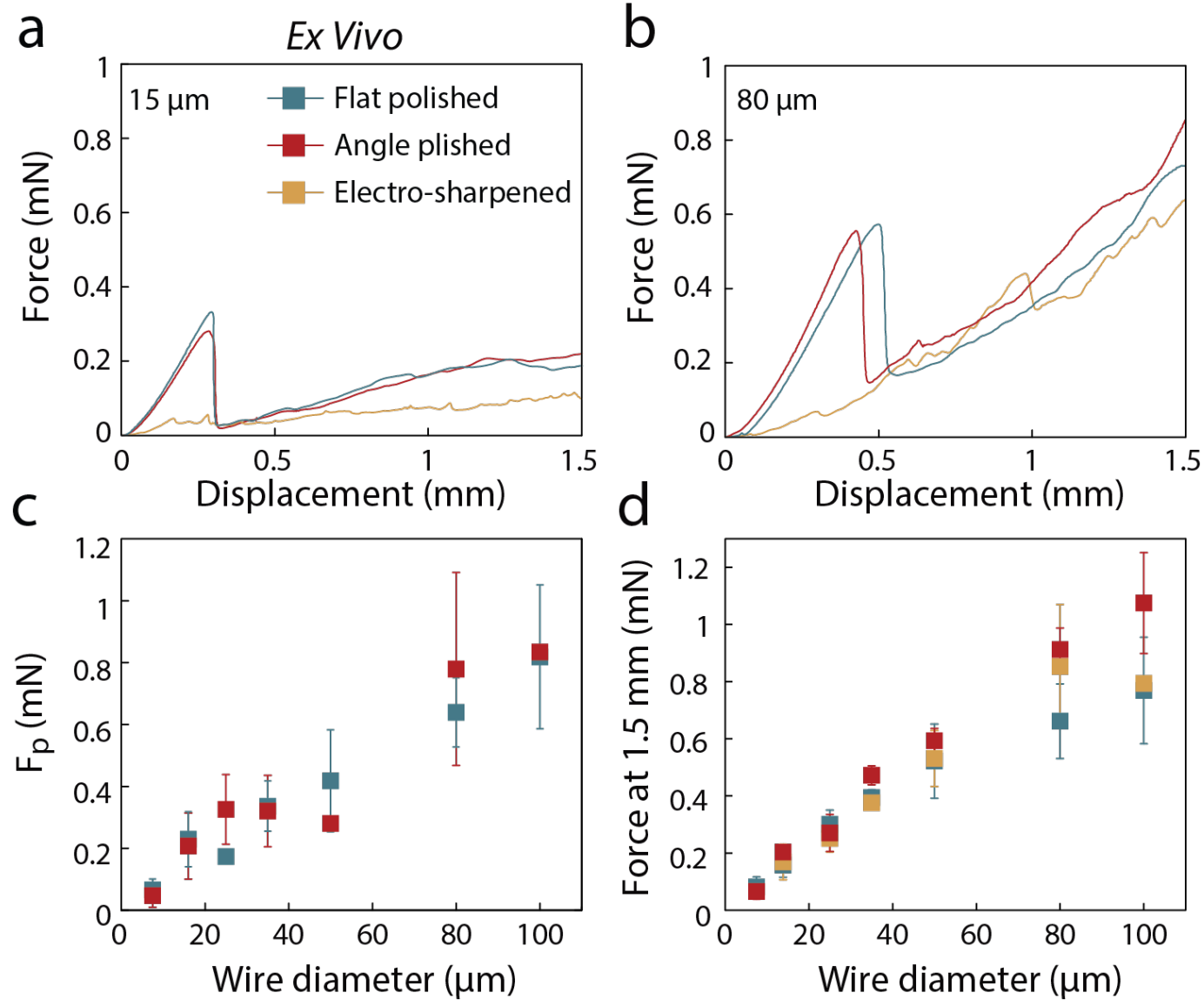

Figure S3 | Ex vivo Tip Geometry dependence

(a,b) Ex vivo force-displacement curves for 3 tip geometries: FP (green), AP (blue), and ES (red), for $15 \mu \mathrm{m}$ diameter (a) and $80 \mu \mathrm{m}$ diameter (b) microwires. (c) Measured puncture force ex vivo as a function of wire diameter for FP and AP wires. (d) Ex vivo force at $1.5 \mathrm{~mm}$ depth as a function of wire diameter for all tip geometries tested. 


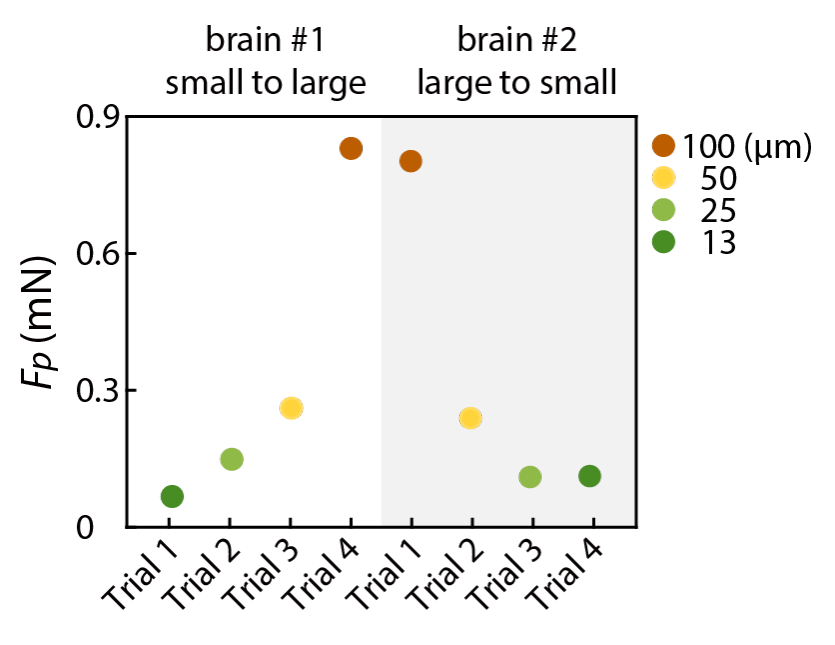

\section{Figure S4 | Reliability of force measurement in ex vivo brain tissue}

In one experiment, two brains were consecutively tested with reverse order of microwire insertion, from small to large diameters, then large to small. The puncture forces $\left(F_{p}\right)$ are shown to be insensitive to the order in which they were tested. These results are consistent with previous work on ex vivo brain in different species ${ }^{17}$.

a)

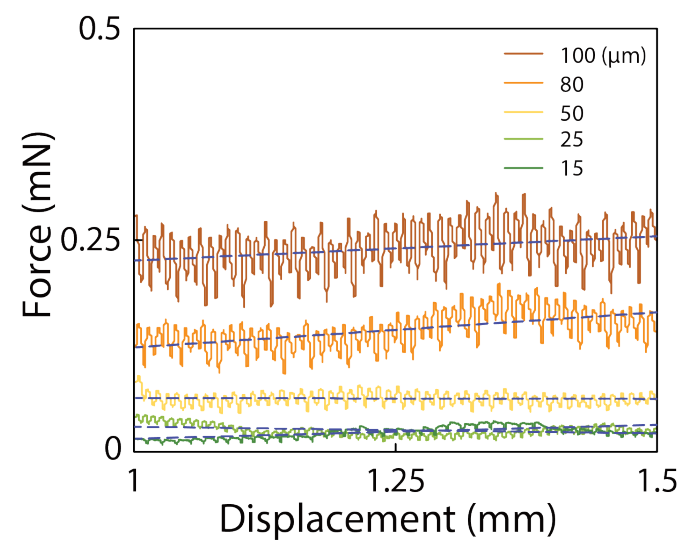

b)

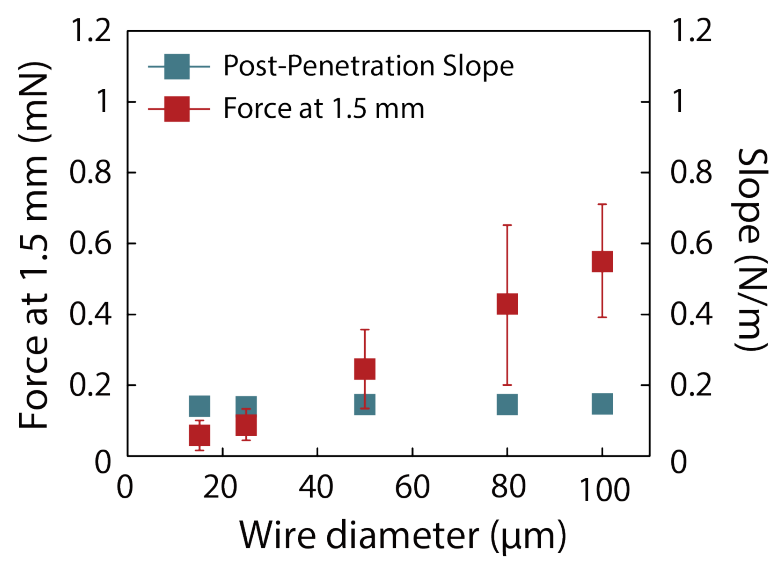

Figure S5 | Past-penetration behavior In Vivo

(a) Force-displacement behaviour of representative traces from 5 different sizes of flat-polished wires. The force oscillates due to breathing and heartbeat but doesn't increase as a function of depth. For larger microwire diameters, the oscillation amplitudes increase. (b) As a function of size, the constant force past-penetration scales with size (red), as do the oscillations from respiration, but the slope of this past-penetration force does not change as a function of size (blue). 


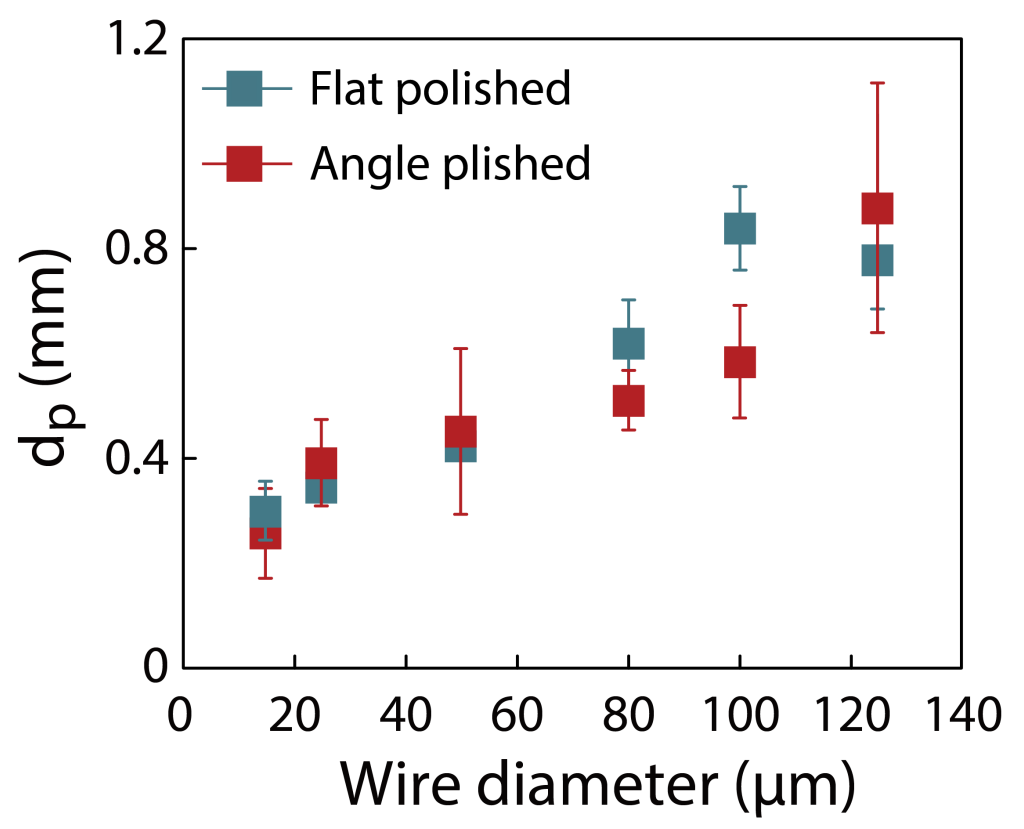

Figure S6 | In Vivo displacement to puncture

Amount of brain compression before puncture as a function of wire diameter for flat and anglepolished electrodes. $d_{p}$ is found to scale linearly with wire diameter for both flat and angle-polished electrodes. 

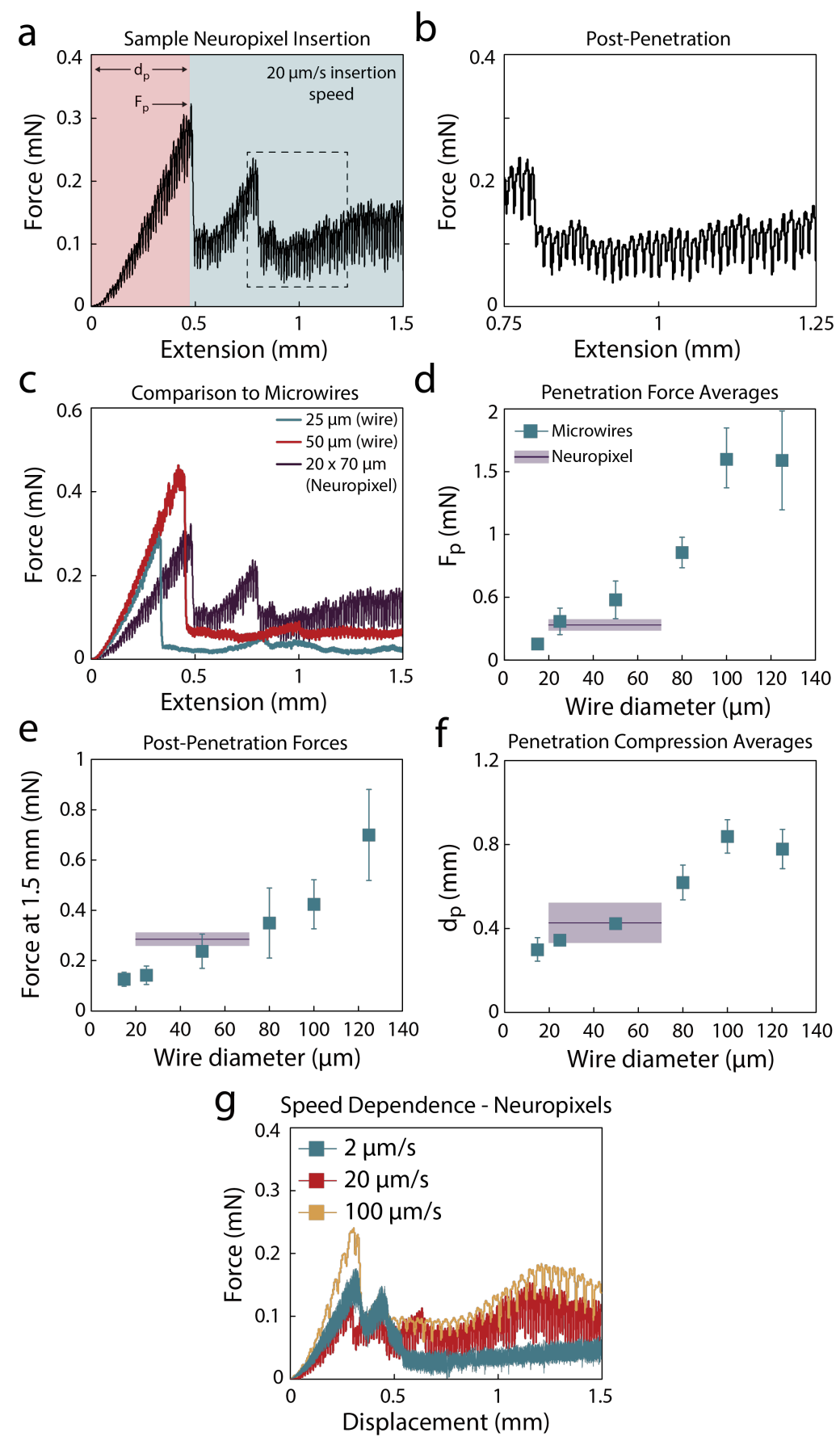

Figure S7 | Neuropixel In Vivo Comparison

(a) Sample Neuropixel inserted in vivo at $20 \mu \mathrm{m} / \mathrm{s}$. Pre-penetration, (b) Zoom-in into the past penetration phase of the Neuropixel insertion. Oscillations due to breathing and heartbeat are consistent with measurements of cylindrical microwires. (c) Neuropixel insertion compared to two representative microwire insertions (50 $\mu \mathrm{m}$ in red and $25 \mu \mathrm{m}$ in blue). The Neuropixel displays a peak penetration force similar to a $25 \mu \mathrm{m}$ microwire (d), but a compression distance and postpenetration forces closer to that of a $50 \mu \mathrm{m}$ microwire (e,f). The size values for Neuropixel in (df) are plotted between $20-70 \mu \mathrm{m}$ to compare with circular microwires. (g) Speed dependence measurements using Neuropixels. No significant differences were observed between $2-100 \mu \mathrm{m} / \mathrm{s}$ insertion speeds. 


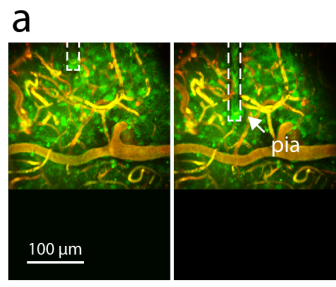

b

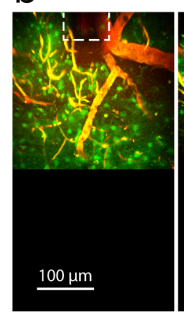

c

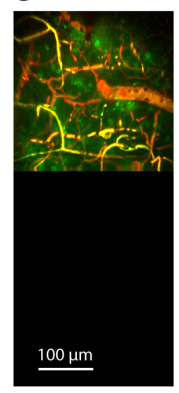

$13 \mu \mathrm{m}$ microwire

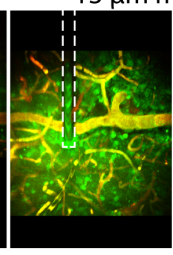

$80 \mu \mathrm{m}$ microwire

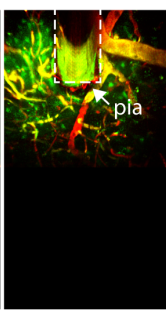

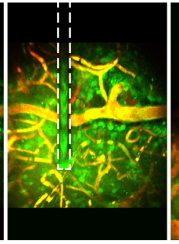
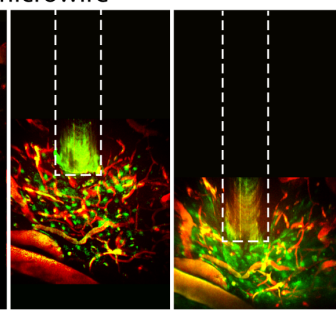

Withdraw

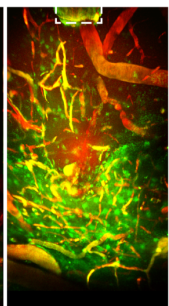

Withdraw
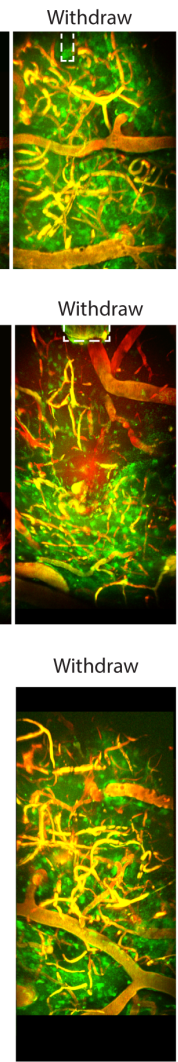

Figure S8 | Two-Photon Microscopy of vascular damage

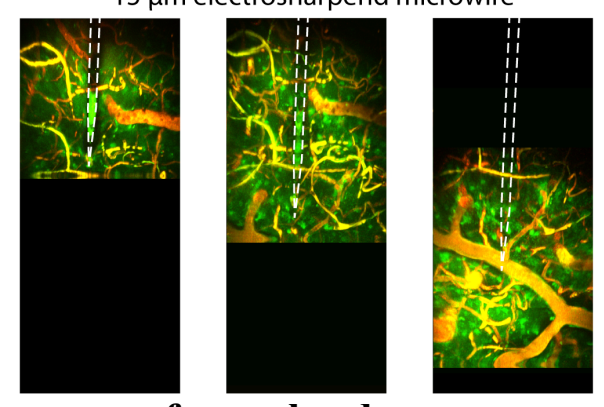

(a-c) Two-photon imaging of $13 \mu \mathrm{m}$ and $80 \mu \mathrm{m}$ flat-polished and $15 \mu \mathrm{m}$ electrosharpened microwire insertion, respectively. Astrocyte-GFP and BV-Rhodamine are used to stain the vasculature and astrocytes. Two-photon image stacks were taken with sequential motion of the microwire, as the imaging took several minutes to acquire, showing a few frames of the insertion process. For the $13 \mu \mathrm{m}$ insertion, the tip of the wire drags a part of the pia surface tissue as the wire inserts. However, no disruption of the tissue is observed as the wire is driven deeper and is finally extracted. In contrast, the $80 \mu \mathrm{m}$ wire (b) causes significant disruption to nearby vasculature and astrocytes. (c) During the insertion of a $15 \mu \mathrm{m}$ electrosharpened microwire, no disruption is observed similar to the flat-polished small microwire (a).

\section{Materials considerations for designing brain-machine interfaces}

The results from this study suggest that smaller electrodes should reduce physiological damage because of reduced tissue compression. At the same time, research had found that lower material stiffness can significantly reduce traumatic response $\mathrm{e}^{9,38}$, thus more flexible, soft probes are preferred. Yet as probes become smaller and/or more flexible they may be too compliant to bear the puncture force, $F_{p}$, and buckle ${ }^{39}$. For example, flexible probes have been inserted through insertion shuttles that provide temporary mechanical stiffness to the probe. Once the probe is successfully in place, the shuttle is removed surgically, or dissolves away ${ }^{3,4,40}$. Unfortunately, these shuttles must be relatively large, which may induce significant primary trauma. 
The interplay between the size of the electrodes and a minimum stiffness to pierce the brain without buckling is given by the buckling force threshold, which must be larger than the force to penetrate the brain. $F_{p}$ was found to be primarily dependent on the diameter and tip geometry of the electrode and is independent of the material modulus. Therefore, the results from this study can predict whether a probe of a given material and size will successfully penetrate the brain before buckling. The theoretical buckling force is calculated using Euler's formula, given by:

$$
\mathrm{F}=\frac{\pi^{2} E I}{(K L)^{2}}
$$

where $E$ is the elastic modulus, $I$ is area moment of inertia, $L$ is the effective length of the wire and $K$ is the effective length factor of the wire $(2.0$ when one end is fixed and the other end free to move laterally).

\section{a}

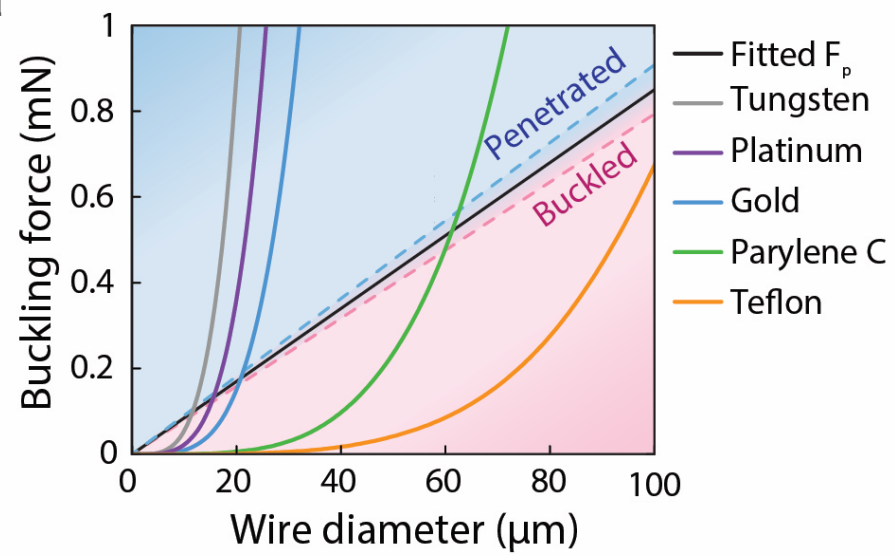

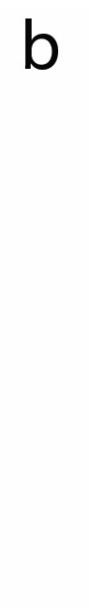

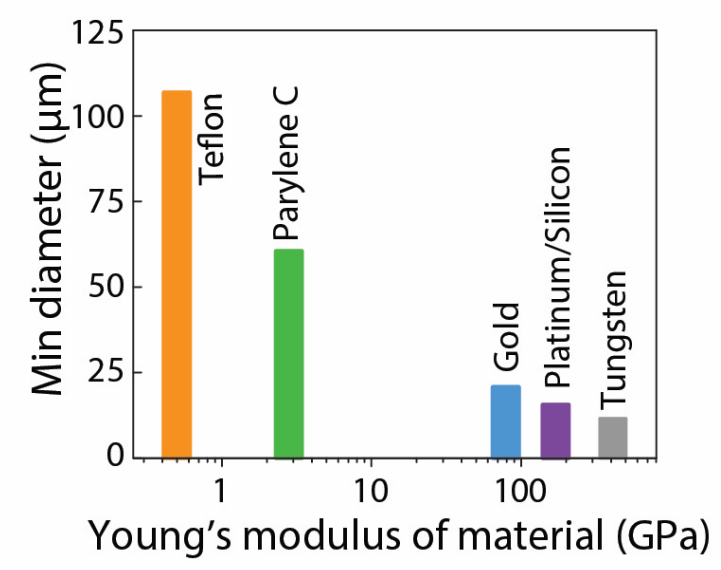

Figure S9. | Probe Material Design Choices

(a) Buckling force curves as a function of wire diameter, calculated using Euler's buckling equation. A $K$ value of 2 is used, assuming one end is rotationally and translationally fixed (distal) and the brain interfacing end is free. The length is chosen to be $L=3.0 \mathrm{~mm}$, a relevant length for brain-penetrating electrodes. Fitted puncture force $\left(F_{p}\right)$ based off of flat-polished microwire dataset. Diameters in the blue region predict successful penetration into brain tissue with a wire of a specific material, while diameters in the red region are calculated to buckle before penetration. (b) The minimum diameter required for a specific material to penetrate into brain tissue, based off of where the theoretical buckling force intersects with the measured puncture force from (a).

Figure S9a plots the buckling force as a function of probe diameter for a variety of materials commonly used as brain penetrating electrodes, assuming a cylindrical wire shape of length $l=3$ $m m$. This length was chosen because it is deep enough to reach most brain areas in mice but may need to be larger to be relevant for deeper brain regions. Plotted on top of the buckling force curves is the measured $F_{p}$ of flat-polished tips with wire diameter (red line). Any probe materials or sizes 
that lie below this line are too compliant and will buckle before penetration, while anything above can penetrate. The minimum wire diameter required for specific materials can be calculated from where the buckling force line intersects with the force to puncture (Fig. S9b). For example, stiff tungsten wires can be as small as $12 \mu \mathrm{m}$ diameter, while gold has to be $>20 \mu \mathrm{m}$ without additional coatings or braces. In contrast, a compliant material like $\mathrm{PaC}$ needs to be $>60 \mu \mathrm{m}$ in diameter to penetrate without buckling. Conversely, these larger electrodes will induce increased initial compression in the brain, thus the benefits of softer, compliant materials must be balanced with larger diameters necessary and increased trauma.

Video S1 | In Vivo Insertion of $15 \mu \mathrm{m}$ Electrosharpened Microwire

Video S2 | Epifluorescence Imaging of $25 \mu \mathrm{m}$ Flat-Polished Microwire with Simultaneous Force Measurement

Video S3 | Epifluorescence Imaging of $80 \mu \mathrm{m}$ Flat-Polished Microwire with Simultaneous Force Measurement 